\title{
An Efficient Combination of Texture and Color Information for Watershed Segmentation
}

\author{
Cyril Meurie, Andrea Cohen, and Yassine Ruichek \\ Systems and Transportation Laboratory, \\ University of Technology of Belfort-Montbliard \\ 13 rue Ernest Thierry-Mieg, 90010 Belfort, France \\ \{cyril.meurie, andrea.cohen, yassine.ruichek\}@utbm.fr
}

\begin{abstract}
A new segmentation technique based on a color watershed using an adaptive combination of color and texture information is proposed on this paper. This information is represented by two morphological gradients, a classical color gradient and a texture gradient based on co-occurrence matrices texture features. The two morphological gradients are then mixed using a gradient component fusion strategy and an adaptive technique to choose the weighting coefficients. The segmentation process is finally performed by applying the watershed algorithm. The obtained results are then evaluated with the MSE for several sets of parameters and color spaces.
\end{abstract}

Keywords: image segmentation, adaptive combination, color, texture, mathematical morphology, co-occurrence matrices.

\section{Introduction}

Many segmentation methods are proposed in the literature. The methods on which this article is based can be grouped into two categories: edge and/or region based segmentation. These methods are usually developed considering specific applications. Therefore, there is no method that can be successfully applied for all applications without parameter redefinition. Generally, images present two important informations which are color and texture. It is, hence, useful to use a segmentation method based on these informations. Angulo 1 proposes a segmentation method combining color and texture information. However, this method involves many parameters, which are difficult to adjust according to the considered application. Considering the popularity of the use of co-occurrence matrices [2] 3] 4] [5] to extract the texture features of an image, we propose a new texture gradient based on co-occurrence matrices. This new texture gradient is then used in a new segmentation technique based on an original and adaptive combination (considering local image content) to take into account both the color and texture gradients. This combination technique has already been tested on previous works ([6] [7])for Angulo's texture gradient [1] for two specific applications. The paper is organized as follows: Section 2 presents the morphological texture and color gradients definition. Section 3 describes the structural gradient

A. Elmoataz et al. (Eds.): ICISP 2010, LNCS 6134, pp. 147-156, 2010.

(C) Springer-Verlag Berlin Heidelberg 2010 
process and the proposed strategy for combining the texture and color gradients. Before concluding, experimental results for the Berkeley Segmentation Dataset and Benchmark are presented for different parameter settings and color spaces in section 4 .

\section{Morphological Texture and Color Gradients Definition}

\section{$2.1 \quad$ Co-occurrence Matrices}

A co-occurrence matrix is, essentially, a two-dimensional histogram of the number of times that pairs of intensity values occur in a given spatial relationship (or translation) 4]. Mathematically, a co-occurrence matrix $C$ is defined over a gray-level image $g$, parameterized by a translation $\boldsymbol{t}$, as:

$$
C_{\boldsymbol{t}}(i, j)=\operatorname{card}\{(x, x+\boldsymbol{t}) \in D: g(x)=i, g(x+\boldsymbol{t})=j\}
$$

where $g(x)$ is the gray level for the pixel $x ; i$ and $j$ are gray levels, and $D$ is the domain of the image.

A co-occurrence matrix describes an image by looking at the relation between neighbor pixels and not each pixel separately. Texture, on the other hand, is a phenomenon associated to a neighborhood of pixels and not to an individual pixel, hence, co-occurrence matrices can be used as a tool for texture description. Nevertheless, the success of this tool highly depends on the spatial relationship (translation vector) chosen. There are several characteristic features of co-occurrence matrices that can be used for textural description. These features summarize the content of the matrices. There is a total of 13 of these features that have been presented by Haralick et al. $[3$. Only three of them are used for extracting feature images on [2]: the entropy, the angular second moment $(A S M)$ and the contrast $(c)$. We will only present and use the $A S M$ and the contrast since, as we proved on our experiences, the entropy does not provide interesting results.

$$
A S M_{\boldsymbol{t}}=\sum_{i=0}^{G-1} \sum_{j=0}^{G-1} C_{\boldsymbol{t}}^{2}(i, j) \text { and } c_{\boldsymbol{t}}=\sum_{i=0}^{G-1} \sum_{j=0}^{G-1}(i-j)^{2} C_{\boldsymbol{t}}(i, j) .
$$

where $G$ represents the number of gray levels taken into account, which is set to 16 for our experiences.

The $A S M$ is a measure of the homogeneity of the texture for the given spatial relationship. Its value is high when the same couple of pixels is found repeatedly throughout the image, resulting in a matrix that has few entries of large magnitude. The contrast feature is a measure of the amount of local variation of the texture according to the spatial relationship. It is high when the matrix presents large terms far away from the diagonal. This means that pixels that are neighbors in terms of vector $\boldsymbol{t}$ have very different intensity values. 


\subsection{Local Analysis}

As presented on the preceding section, co-occurrence matrices can be used to obtain texture features. Nevertheless, they are computed globally for the entire image, while the expectation is to obtain more than one texture per image. Indeed, the analysis should be able to be applied for each pixel of a color image $f$. To solve this problem, a co-occurrence matrix is computed locally for each pixel. This can be done by calculating $C$ on a window centered on each pixel $x$. In this way, both measures ( $A S M$ and contrast) can be computed for each pixel of the image. It is defined as the local ASM image associated to $t$ the image that groups the $A S M$ measures associated to the translation $\boldsymbol{t}$ of each pixel of the image. The same concept can be applied to the contrast, resulting in a local contrast image associated to $\boldsymbol{t}$. In a $\boldsymbol{t}$-oriented local ASM image (resp. contrast image), pixels associated to a texture that's homogeneous in the direction and size of $\boldsymbol{t}$ (or that presents a lot of contrast for the contrast image) are affected with a high value. The images obtained by this procedure are then normalized. Since co-occurrence matrices can only be computed over a gray-level image, they are computed seperately for each color plane of the input image $f$ in the RGB color space. This results in three $\boldsymbol{t}$-oriented local ASM images (resp. contrast images) per color image $f$ per translation vector $\boldsymbol{t}$. By using a set of vectors with different sizes and orientations, one can obtain texture feature images that are responsive to a number of textures with different orientations. The chosen set of vectors must cover the whole spectre of textures present in the image.

\subsection{Morphological Texture and Color Gradients Computation}

A morphological gradient is defined, over a gray-level image $g$, as the residue of the dilatation and the erosion (usually computed with a structuring element of size 1):

$$
Q(g(x))=\delta_{\beta}(g(x))-\epsilon_{\beta}(g(x)) .
$$

where $\beta$ represents the structuring element (normally a disc of radius 1 ). If $f$ is a color image, then $\delta(f(x))$ and $\epsilon(f(x))$ are color vectors, and the classical morphological color gradient $f$ is given by the preceding formula using a conditional (also called lexicographic) vector ordering [8] [9] [10] [11. The morphological texture gradient is defined using references to the concept of texture feature images. This concept may refere to the ASM local images for the computation of an ASM texture gradient, and to contrast local images for the computation of a contrast texture gradient previously presented. Each one of these texture feature images is refered to as $t_{k}$. The texture gradient of the image is finally defined in reference to the morphological gradient of each $t_{k}$ :

$$
Q_{t e x}(f(x))=\bigvee_{k \in K}\left[Q\left(t_{k}(x)\right)\right]
$$

where $K$ is a set of vectors with different sizes and orientations (such as those presented on table 1). The process to compute a morphological texture gradient 
Table 1. Tested sets of vectors in co-occurrence matrices

\begin{tabular}{|c||c||c||c|}
\hline Set & Nb of vectors & Vectors' sizes & Vectors' orientation \\
\hline 1 & 4 & 2 & $0,45,90,135$ \\
\hline 2 & 6 & 2 & $0,30,60,90,120,150$ \\
\hline 3 & 8 & 4 & $0,30,45,60,90,120,135,150$ \\
\hline 4 & 10 & 4 & $0,30,37,45,60,90,120,127,135,150$ \\
\hline 5 & 16 & $2-4$ & $0,30,45,60,90,120,135,150$ \\
\hline 6 & 8 & 8 & $0,30,45,60,90,120,135,150$ \\
\hline
\end{tabular}

for both the contrast and the $A S M$ measures is illustrated on figure 1, Other definitions may be applied for the computation of the texture gradient, such as an euclidian gradient. However, the supremum gradient is simpler to compute and has been proved to be as efficient in [1. It should be pointed out that both resulting texture gradients ( $A S M$ and contrast) are gray level images.

\section{Structural Gradient Definition}

In order to achieve a robust and reliable segmentation, it is very useful to use both texture and color information. The main idea is to produce a structural gradient by combining the texture and color gradients. The problem is the fact that the color gradient is a color image while the texture gradient is a gray level image. To solve this problem, the proposed method starts by decomposing the color gradient image $Q_{c o l}$ to obtain its three components which are $Q_{c o l}^{R}$, $Q_{c o l}^{G}$ and $Q_{c o l}^{B}$. In the next step, each component of the color gradient image is combined with the texture gradient image $Q_{\text {tex }}$, where $Q_{\text {tex }}$ may refer to either a contrast-based gradient or a ASM-based gradient. This operation produces three gray levels images $Q^{R}, Q^{G}$ and $Q^{B}$ :

$$
\left\{\begin{array}{c}
Q^{R}=Q_{\text {col }}^{R} \otimes Q_{t e x} \\
Q^{G}=Q_{c o l}^{G} \otimes Q_{t e x} \\
Q^{B}=Q_{c o l}^{B} \otimes Q_{t e x}
\end{array}\right.
$$

$Q^{R}, Q^{G}$ and $Q^{B}$ can be interpreted as the color planes of a new color image, which is proposed to define the needed structural gradient. In other words, the structural gradient $Q$ is defined as a color image with $Q^{R}$ as the red component, $Q^{G}$ as the green component, and $Q^{B}$ as the blue component. This combination approach is suitable because, not only color information is preserved, but also the texture information is added to each color component. Indeed, if we asume that texture is not a color phenomenon, then it is supposed to affect all colors equally. Other strategies involving the fusion of the color gradient into a gray level image have been discarded since they give very bad results. The operator $\otimes$ represents the combination of two gray level images: a component of the color gradient image $q$ and the texture gradient image $r$. Three techniques are used: 

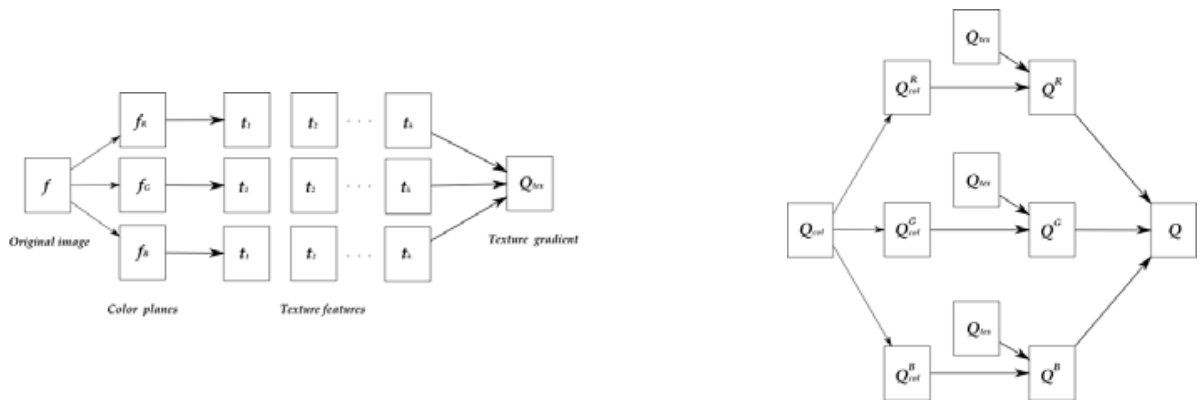

Fig. 1. Illustration of the structural gradient computation process (left: texture gradient computation; right: structural gradient computation)

a fixed combination, an adaptive combination and a supremum combination. An illustration of this process is given in figure 1, Let $h$ be the output of the combination process which is applied for each pixel.

\subsection{Fixed Combination}

The fixed combination is defined as a barycentric sum of the color and the texture gradient images. It uses a global weighting coefficient referred to as $\alpha$ :

$$
h(p)=\alpha q(p)+(1-\alpha) \times r(p)
$$

where $\alpha$ is a constant coefficient taking its value in $[0 ; 1]$. The combination technique is not generally suitable, due to the coefficient $\alpha$, which is constant for the entire image. Indeed, one may need to give priority to color or texture according to their importance in the different zones of the image. This technique requires manual adjustment of the coefficient $\alpha$ according to the content of the image.

\subsection{Adaptive Combination}

The proposed adaptive combination strategy uses a modular combination of texture and color gradients according to the content of the image. This implies two advantages: first, it gives priority to the most important information (the color or the texture) for a given pixel. Second, it constitutes an automatic method, which can be performed for all types of images. This technique has been tested for a different texture gradient in [6], [7. The adaptive combination is expressed as follows:

$$
h(p)=\alpha_{p} q(p)+\left(1-\alpha_{p}\right) \times r(p) \text { and } \alpha_{p}=\frac{q(p)}{q(p)+r(p)} .
$$

$\alpha_{p}$ is a coefficient taking its value in $[0 ; 1]$. It is calculated for each pixel $p$ in order to give a high weight to the image that provides the most important information for the pixel. In other words, $\alpha_{p}$ is high if the information is more important for $f$ than for $g$ (and vice-versa). 


\subsection{Supremum Combination}

Using the same principle as in the adaptive strategy, the supremum combination is sensibly different. There is actually no combination at pixel level. Indeed, for a given pixel, the modular gradient is either a copy of the color gradient or the texture gradient depending on which one of them provides the greatest amount of information (the supremum). This combination has the same advantages as the adaptive one.

$$
h(p)=\left\{\begin{array}{l}
q(p), \text { si } q(p) \geq r(p) \\
r(p), \text { si } q(p)>r(p)
\end{array}\right.
$$

\section{Experimental Results}

In this section, we evaluate the proposed approach for image segmentation by combination of color and texture information using eight images of the Berkeley Segmentation Dataset and Benchmark (BSDB). To analyse the different segmentation results, we use the Mean Square Error $(M S E)$ which does not require a reference segmentation. The influence of the color space is also studied for six color spaces (RGB, HSL, $\left.I_{1} I_{2} I_{3}, Y C_{b} C_{r}, Y C h_{1} C h_{2}, L^{*} a^{*} b^{*}\right)$, each one of them belonging to one of the six main color space families described in the literature [12. Segmentation is achieved by performing a watershed algorithm [13] [14. This algorithm allows to segment an image into homogeneous regions from a seeds image (markers) and a potential image (gradient). The seeds image corresponds to $5 \%$ of local minimum values of the original image, while the gradient image corresponds to the structural gradient presented on the preceding section. For better vizualisation, we only present the results obtained with the best parameter settings (figure 2): on the top of the figure, average results for the best window sizes for RGB colors space are presented for each each collection of table 1. On the bottom, we illustrate the results corresponding to the best collection for each color space for the same window sizes. The different curves on the graphs correspond to several structural gradients computed for the contrast-based texture gradient. Results for the ASM-based texture gradient are not presented since they are not satisfactory enough.

The worse segmentation results are obtained with a window of size 16 (except for the $\alpha=0.7$ fixed combination for which the results are almost unchanged regardless the windows size). This is why they are not presented in figure 2 The proposed adaptive combination technique always gets the second or the third place if not the first. In average, a fixed combination of $\alpha=0.7$ gives the best results but we should not forget that this choice of $\alpha$ was found after a grat number of experiments and it is not guaranteed to work for a different set of images. This is not the case for the proposed adaptive method. In $R G B$ color space, the best results are obtained: 1 / for the proposed adaptive combination with a window size of 4 and the collection 1 (figure2 top-left); 2 / for the combination $\alpha=0.7$ with a window of size 6 and the collection 5 (figure 2 top-right). To analyze the influence of the color space on the segmentation results, these two window sizes ( 4 and 6 ) are kept 

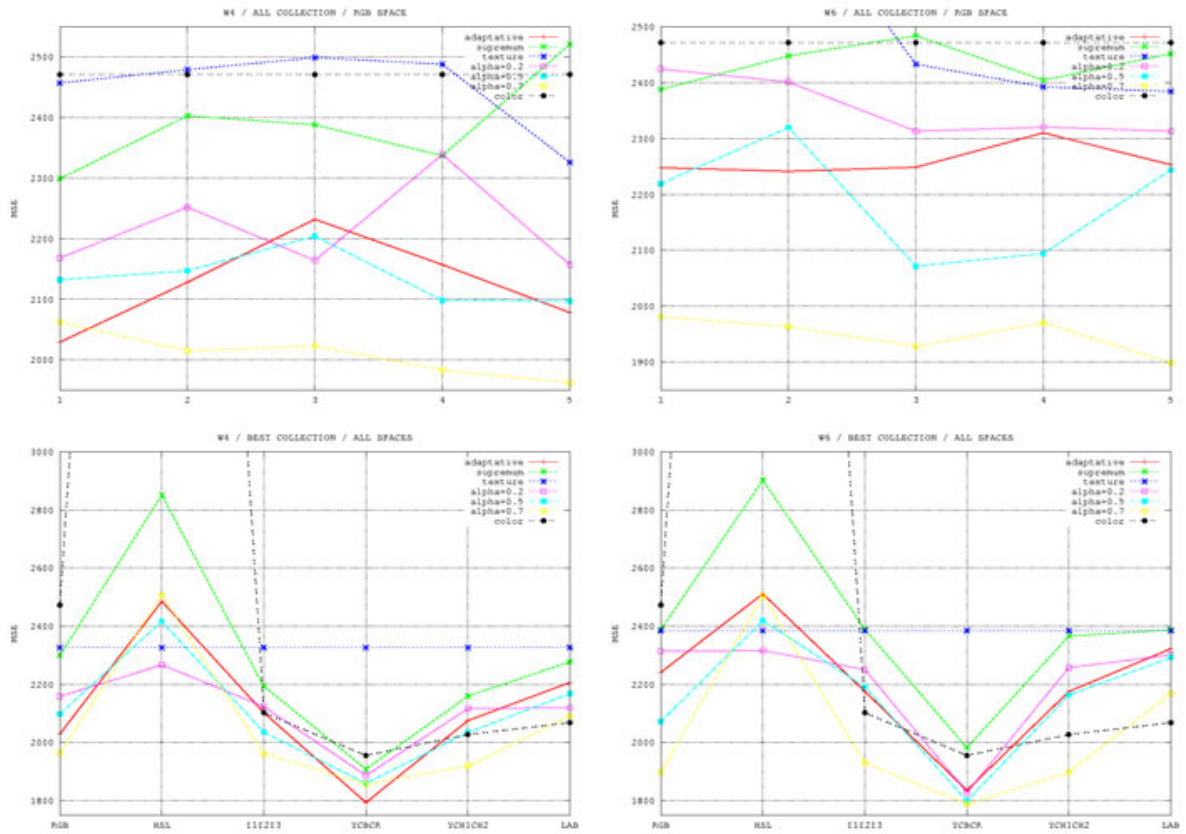

Fig. 2. Evaluation of segmentation results in RGB color space for all collections (top) and in different color space for the best collection (bottom), with a window size of 4 (left) and a window size of 6 (right).
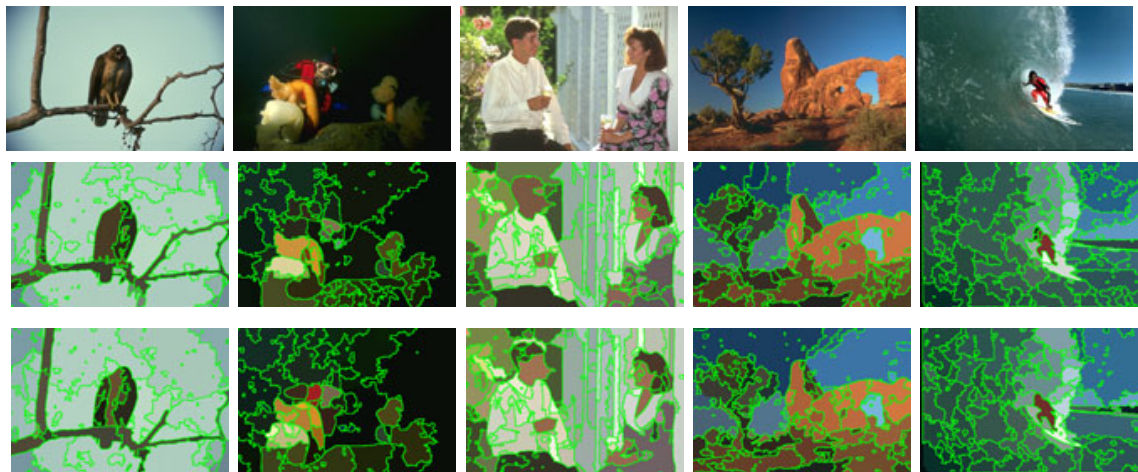

Fig. 3. Segmented images with the best gradient combinations in Berkeley Segmentation Database and Benchmark (top to bottom : initial image, segmented images with combination $\alpha=0.7$, segmented image with adaptive combination) 

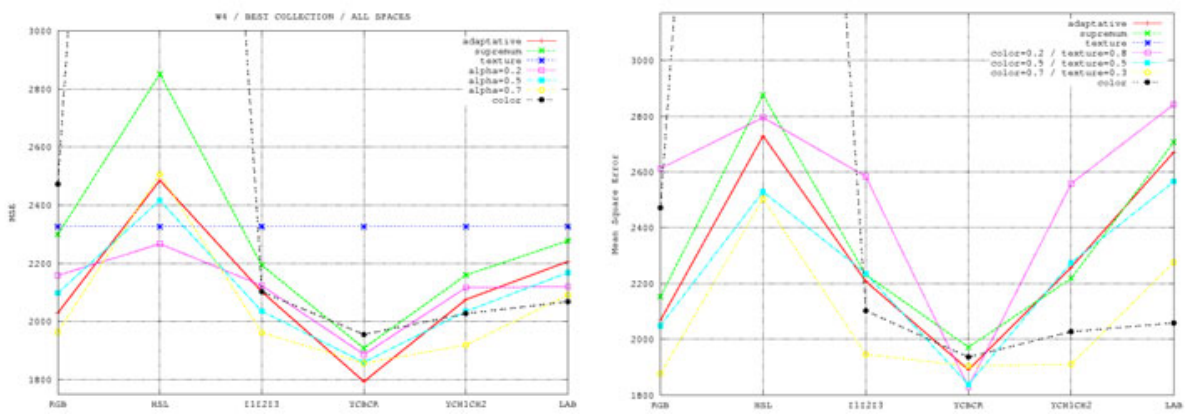

Fig. 4. Evaluation of segmentation results in different color spaces for the best collection and window size (left), evaluation of segmentation results for previous works in different color spaces and with the best parameters (right)

and tested for all collections in table1. We can conclude that the best results are obtained with the proposed adaptive method (with a window of size 4) and for the $\alpha=0.7$ fixed combination (with a window of size 6) in $Y C_{b} C_{r}$ color space. For the $\alpha=0.7$ fixed combination, the best results are obtained for collection 4 . This emphasizes the importance of the choice of the vector set, which can vary according to the application. For the proposed adaptive combination, the best results are obtained for collection 2. Nevertheless, it is important to point out that the proposed adaptive method is also the best regardless of the collection chosen (and no parameter settings are required as opposed to the fixed method). In figure 3, we illustrate the segmentation results for $Y C_{b} C_{r}$ for the two best combinations (ie. $\alpha=0.7$ and adaptive). Even if the results are similar for the fourth image, we can notice that the proposed method provides better results for the four other images. For example, image 1 shows a better segmentation of the bird's parts, the seaweed and diver are better extracted on image 2, the man's hair and the woman's arm are well segmented on image 3 and the surfboard is better extracted on image 5 .

The method has been compared to previous works (which replace the texture gradient computation with a morphological texture gradient, [6] [7]). The evaluation results for both methods are presented on figure 4. As we can see, both methods present the best results in $Y C_{b} C_{r}$ color space. However, the previous morphological gradient only achieves a $M S E$ of 1900 with the adaptive combination while the present method achieves a $M S E$ of 1800 for this combination. This beats the best results obtained by the older method, thus demonstrating the interest of developing this new approach.

\section{Conclusion}

A new segmentation technique based on the watershed algorithm by combination of both color and texture information is proposed. This information is represented by two morphological gradients, a classical color gradient and a texture gradient based on co-occurrence matrices texture features. These two morphological gradients are then mixed using a gradient component fusion strategy. 
The originality of the method is that it takes into account local image content by automatically computing the weighting coefficients for color and texture. The classical technique uses a fixed combination and requires the choice of the collection and a manual adjustment of the weighting coefficients which are global for all the pixels of the image. On the contrary, the proposed method does not require any parameters settings. For the $\mathrm{BSDB}$, the best results are obtained with the proposed adaptive method in the $Y C_{b} C_{r}$ color space (with a window of size 4 which is a parameter for the calculation of the texture gradient). The segmentation results for the proposed method are very satisfactory considering those obtained by other methods which, in addition, present the disadvantage of requiring a parameter adjustment.

Perspectives concern the reduction of the execution time in order to be able to use the proposed method on a real time application. Future works will also focus on the computation of the texture gradient in different color spaces as well as the definition of a texture gradient as a color image instead of a gray level image.

\section{References}

1. Angulo, J.: Morphological texture gradients. Application to colour+texture watershed segmentation. In: Proc. of the 8th International Symposium on Mathematical Morphology, October 2007, pp. 399-410 (2007)

2. Kruizinga, P., Petkov, N.: Nonlinear operator for oriented texture. IEEE Transactions on image processing 8(10), 1395-1407 (1999)

3. Haralick, R.M., Shanmugan, K., Dinstein, I.: Textural features for image classification. IEEE Trans. On Systems, Man, and Cybernetics 3(6), 610-621 (1973)

4. Zucker, S.W., Terzopoulos, D.: Finding structure in co-occurrence matrices for texture analysis. Computer Graphics and Image Processing 12, 286-308 (1980)

5. Baraldi, A., Parmiggiani, F.: An investigation of the textural characteristics associated with gray level cooccurrence matrix statistical parameters. IEEE Trans. On Geoscience and Remote Sensing 33(2) (March 1995)

6. Cohen, A., Meurie, C., Ruichek, Y., Marais, J., Flancquart, A.: Quantification of gnss signals accuracy: an image segmentation method for estimating the percentage of sky. In: IEEE International Conference on Vehicular Electronics and Safety (ICVES), November 2009, pp. 40-45 (2009)

7. Meurie, C., Ruichek, Y., Cohen, A., Marais, J.: An hybrid an adaptive segmentation method using color and textural information. In: IS\&T/SPIE, Electronic Imaging 2010 - Image Processing: Machine Vision Applications III (January 2010)

8. Meurie, C.: Segmentation of color images by pixels classification and hierarchy of partitions. Ph.D. dissertation, University of Caen Basse-Normandie, Caen, France (October 2005)

9. Lezoray, O., Meurie, C., Elmoataz, A.: A graph approach to color mathematical morphology. In: IEEE Symposium on Signal Processing and Information Technology (ISSPIT), pp. 856-861 (2005)

10. Lezoray, O., Meurie, C., Elmoataz, A.: Graph-based ordering scheme for color image filtering. International Journal of Image and Graphics 8(3), 473-493 (2008) 
11. Aptoula, E., Lefevre, S.: A comparative study on multivariate mathematical morphology. Pattern Recognition 40(11), 2914-2929 (2007)

12. Vandenbroucke, N.: Segmentation d'images par classification de pixels dans des espaces d'attributs colorimétriques adaptés. Ph.D. dissertation, University of Lille 1 (December 2000)

13. Vincent, L., Soille, P.: Watersheds in digital spaces: an efficient algorithm based on immersions simulations. IEEE Trans. On Pattern Analysis and Machine Intelligence (PAMI) 13(16), 583-598 (1991)

14. Beucher, S.: Watershed, hierarchical segmentation and waterfall algorithm. Mathematical morphology and its applications to image and signal processing, 69-76 (1994) 